\title{
CDISC Alzheimer's Therapeutic Area User Guide Version 2.0
}

National Cancer Institute

\section{Source}

National Cancer Institute. CDISC Alzheimer's Therapeutic Area User Guide Version 2.0.

NCI Thesaurus. Code C161459.

The 2.0 version of the CDISC Alzheimer's therapeutic area user guide. 